\title{
Refractive index dynamics of quantum dot based waveguide electroabsorbers
}

\author{
Tomasz Piwonski, ${ }^{1}$ Jaroslaw Pulka, ${ }^{1}$ Evgeny A. Viktorov, ${ }^{1}$ Guillaume Huyet, ${ }^{1}$ and \\ John Houlihan ${ }^{1,2, a)}$ \\ ${ }^{1}$ Tyndall National Institute, Lee Maltings, Cork, Ireland and Department of Applied Physics, \\ Cork Institute of Technology, Ireland \\ ${ }^{2}$ Department of Computing, Maths and Physics, Waterford Institute of Technology, Waterford, Ireland
}

(Received 27 June 2010; accepted 14 July 2010; published online 4 August 2010)

\begin{abstract}
The refractive index dynamics of InAs/GaAs quantum dot based waveguide absorbers is studied using heterodyne pump-probe measurements. Absorption reduction due to the pump can be accompanied by either positive or negative refractive index changes depending on the wavelength used. This change in sign of the phase amplitude coupling can be understood by considering the atomlike nature of the quantum dot transitions involved. (C) 2010 American Institute of Physics. [doi:10.1063/1.3476347]
\end{abstract}

The study of self assembled quantum dot (QD) based optical materials and devices was originally motivated by a desire for atomlike optical properties in a compact solid state structure. If applied to semiconductor lasers, researchers envisioned low phase-amplitude coupling, very high differential gain, and temperature insensitive operation, however these expectations have been tempered by the realization that complicating factors exist such as lack of size control, the asymmetry in electron and hole effective mass and the effects of the host matrix. ${ }^{1}$

Nonetheless, niche application areas have been identified where QD based structures are outperforming their bulk and quantum well counterparts, for example monolithic mode locked lasers. ${ }^{2}$ Recent reports include very low pulse to pulse jitter for both harmonically mode locked ${ }^{3}$ and dual mode injected devices, ${ }^{4}$ where the former also demonstrated parameter insensitive dispersion compensated pulses of $700 \mathrm{fs}$ duration over a locking range of $100 \mathrm{MHZ}$, while the latter showed spectral tuning over $10 \mathrm{~nm}$ and large reductions in time bandwidth product. Additionally, interesting features such as wavelength bistability with absorber bias voltage have been reported but remain unexplained. ${ }^{5}$ Important related applications include electroabsorption modulators ${ }^{6}$ and saturable absorber mirrors. ${ }^{7}$ Understanding the ultrafast gain and refractive index dynamics in these novel materials is key to successful exploitation of these devices. In this paper, we measure the refractive index dynamics in a waveguide absorber and show that the pump induced refractive index changes can be either positive or negative depending on wavelength.

The QD waveguide absorber device studied was $1 \mathrm{~mm}$ long, had $4 \mu$ width ridges together with tilted, antireflection coated facets. It was fabricated from material that included six stacks of InAs/GaAs QDs in a dots-in-a-well structure, grown by Zia Inc. (see Ref. 8 for further details of the material). The absorption spectra under reversed biased conditions were measured using a continuous tunable source and

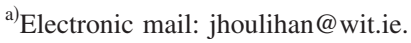

are displayed in Fig. 1. The inhomogeneously broadened ground state transition is clearly visible around $1320 \mathrm{~nm}$ at $0 \mathrm{~V}$. As the reverse voltage is increased, the peak is shifted to longer wavelengths due to the quantum confined Stark effect while the absorption reduces due to increased spatial separation of electron and hole wave functions with increasing electric field (see Ref. 9 for further explanation of a similar structure).

To study the dynamics of the absorption and refractive index, heterodyne pump probe measurements were carried out for a variety of wavelengths and reverse voltages, using pump and probe pulses at the same wavelength. Using this technique, the ultrafast changes and subsequent recovery in absorption (and refractive index) due to an incident pump pulse can be inferred by measuring the amplitude (and phase) of a probe pulse whose delay with respect to the pump pulse is varied (see Ref. 10 for further experimental details). As reported previously, the absorption recovery dynamics can be classified into two regimes depending on reverse voltage. At low voltages, carrier dynamics within the dot together with thermionic emission are the important mechanisms while at higher voltages, tunneling processes

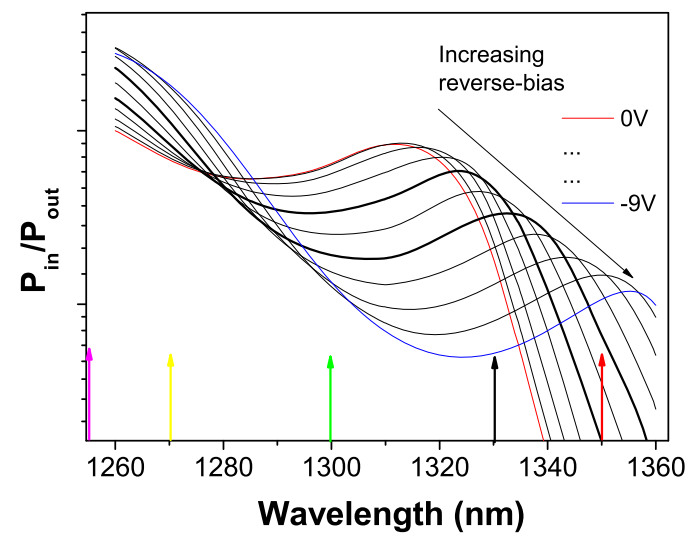

FIG. 1. (Color online) Absorption spectra of the QD waveguide absorber as a function of reverse bias voltage. Wavelengths (and voltages) at which pump probe measurements were carried out are indicated by arrows (and bold lines). In each case, the device was pumped and probed at the same wavelength. 

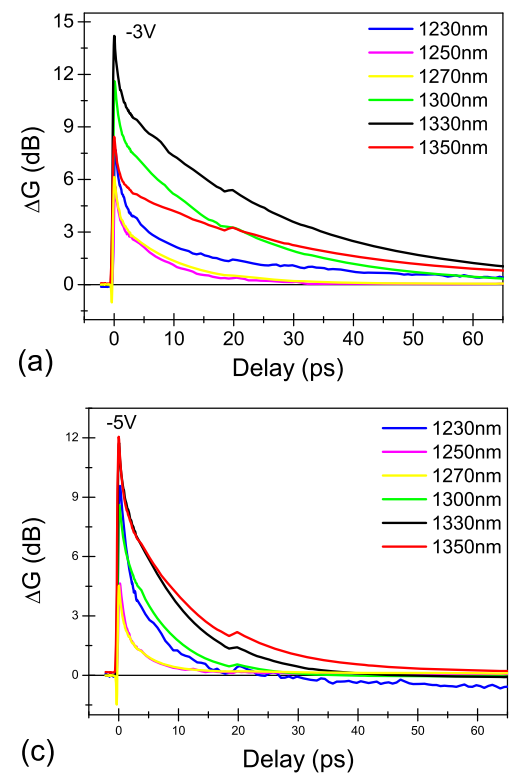

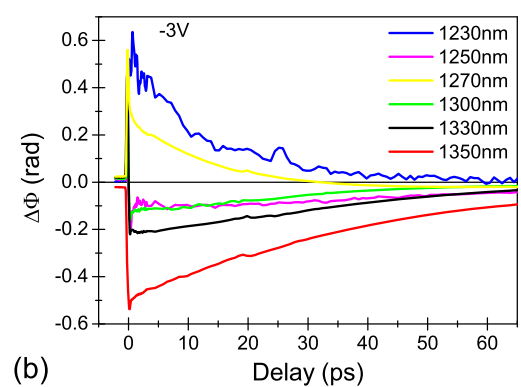

(b)

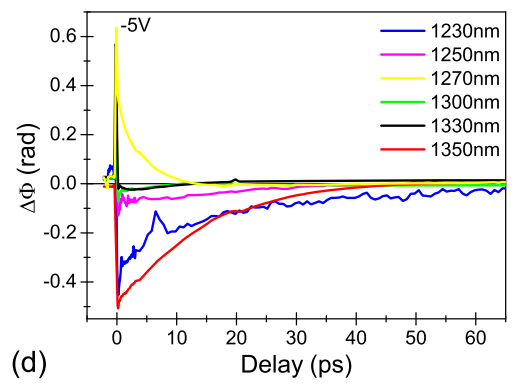

FIG. 2. (Color online) Absorption and phase recovery dynamics taken at reverse voltages of $-3 \mathrm{~V}$ and $-5 \mathrm{~V}$. The laser's repetition rate was $80 \mathrm{MHz}$. dominate. ${ }^{11}$ In this paper, we concentrate on the low voltage regime.

Presented in Fig. 2 are the absorption and phase recovery dynamics at reverse bias voltages of $-3 \mathrm{~V}$ and $-5 \mathrm{~V}$, respectively, carried out at various wavelengths with a pump power of $0.5 \mathrm{~mW}$. As is apparent from the absorption data, the shape of the absorption recovery does not show large changes with wavelength for either 3 or $-5 \mathrm{~V}$ cases. The main variations are twofold. First, there is some variation in the absorption bleaching over the wavelength range due to the shape of the absorption spectrum (Fig. 1), and second, there is a faster absorption recovery around excited state wavelengths due to Auger dominated carrier dynamics for excited state carriers. ${ }^{12}$

In contrast, the phase change associated with pump induced absorption bleaching shows a large variation with wavelength at both 3 and $-5 \mathrm{~V}$ and can be either positive or negative. For both reverse bias cases, the magnitude of the initial phase change due to absorption bleaching is large at either end of the spectral region, and much smaller close to the inhomogeneously broadened ground and excited state absorption maxima. In between these absorption maxima (1270 $\mathrm{nm}$ case), the magnitude is increased to a level similar to that occurring at either end of the spectral region. The sign of the phase change also varies with wavelength and switches a number of times over the spectral region studied in both reverse bias cases. The region where the sign change occurs is close to ground and excited absorption maxima in both voltage cases. Also, when the magnitude of the initial phase change is large, the shape of the phase recovery is very similar to that of the gain recovery, containing two stages of recovery, ${ }^{12}$ where the magnitude of the initial phase change seems smaller, in the vicinity of the ground and excited absorption peaks, the apparent recovery time is much longer and will be discussed in a later section.

This behavior is in contrast to the forward biased phase measurements reported previously, ${ }^{13}$ in which the phase recovery dynamics exhibited much larger contributions at long timescales that implied the role of higher order dot and wetting layer carrier populations in the pump induced phase change and recovery. In our reversed bias case, the lack of large, long timescale components, and similarity of phase and gain dynamics in some spectral regions would suggest against such a strong role for nonresonant carriers in this case. Also, the sign of the pump induced phase change across the ground state is consistent with that expected from a simple artificial atom.

In order to further investigate the spectral dependence of the phase dynamics and the impact of the relatively wide spectrum of our ultrafast pulses $(20 \mathrm{~nm})$, a bandpass filter (bandwidth $10 \mathrm{~nm}$ ) was inserted just before the final detector to examine the phase dynamics for different groups of modes and compare those to that measured for the entire pulse. The results of such a measurement are shown in Fig. 3 at two different wavelengths, for a reverse voltage of $-3 \mathrm{~V}$.

As is clear from panels (a) and (c) in Fig. 3, apart from small amplitude changes, filtering the pulse before detection does not have a large effect on the measured absorption dynamics. However, in the case of the phase dynamics a much different situation exists. At the maximum of the ground state absorption [1320 nm, panel (b)], the initial phase change for the total pulse is small and negative while the filtered cases show different behaviors depending on which spectral region of the pulse is examined. For the central portion the phase change is small and positive, for the short wavelength side, the change is large and positive and for the long wavelength side, the change is large and negative. Thus, the phase of the total pulse contains canceling contributions from either side of the ground state maximum absorption, across both stages of phase dynamics.

At a longer central wavelength of $1340 \mathrm{~nm}$ [panel (d)], the initial phase shift also shows a variation across the pulse spectrum, containing large contributions from central and longer wavelengths and a very small contribution from shorter wavelengths (toward the peak absorption), resulting in an intermediate phase change for the total pulse. Such behavior is consistent with the artificial atom picture of the QD. In the low voltage reverse bias case, nonresonant wetting layer states do not play a significant role in the phase 


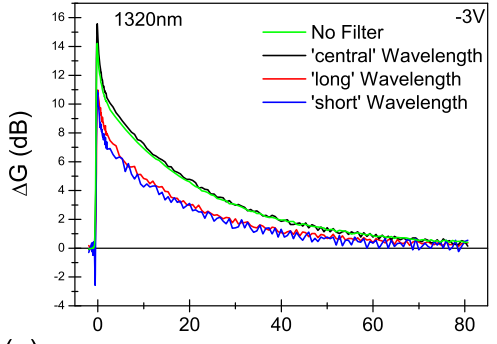

(a)

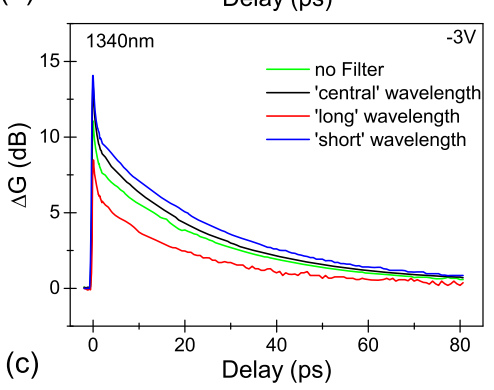

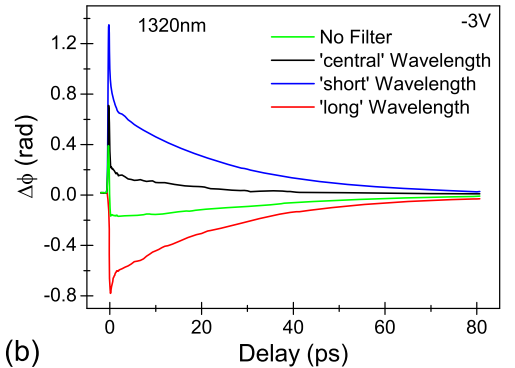

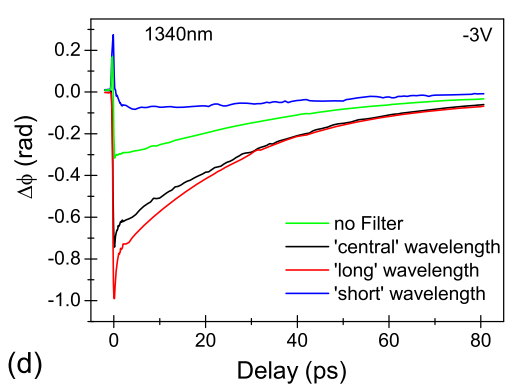

FIG. 3. (Color online) The effect of spectral filtering of the signal just before the final detector for two different central wavelengths at a reverse voltage of $-3 \mathrm{~V}$. Different wavelength groups are selected by rotating the bandpass filter. change associated with pump induced absorption bleaching, in contrast to the forward biased amplifying regime of operation.

To summarize, a pump probe investigation of the refractive index dynamics in a QD waveguide absorber has been carried out at various reverse voltage levels and wavelengths. In particular, the sign of the refractive index dynamics depends on the sign of detuning from the local absorption maximum with much smaller refractive index changes occurring at maximum due to cancellation between positive and negative detuned frequencies. As a result, the alpha-factor for the absorber can vary significantly with wavelength and can be positive or negative. This behavior, unique to QD based absorbers, is due to their atomlike transitions and could explain some features that have been reported in QD based mode-locked lasers such as the wavelength bistability with absorber voltage reported in Ref. 5. Such sign changes do not occur in the forward biased gain regime due the presence of significant carrier populations in higher-lying dot and wetting layer states.

The authors would like to gratefully acknowledge discussions with R. J. Manning. This study has been supported by Science Foundation Ireland under Contract No. SFI 07/ IN.1/I929, the INSPIRE Programme, funded by the Irish Government's Programme for Research in Third Level Institutions, Cycle 4, National Development Plan 2007-2013, and the Institute of Technology Ireland's Strand I Programme and the Tyndall National Access Program.

${ }^{1}$ D. Bimberg, M. Grundmann, and N. N. Ledentsov, Quantum Dot Heterostructures (Wiley, New York, 1999).

${ }^{2}$ E. U. Rafailov, M. A. Cataluna, and W. Sibbett, Nat. Photonics 1, 395 (2007).

${ }^{3}$ H. Schmeckebier, G. Fiol, C. Meuer, D. Arsenijevic, and D. Bimberg, Opt. Express 18, 3415 (2010).

${ }^{4}$ T. Habruseva, S. O’Donoghue, N. Rebrova, D. A. Reid, L. P. Barry, S. P. Hegarty, D. Rachinskii, and G. Huyet, IEEE Photon. Technol. Lett. 22, 359 (2010).

${ }^{5}$ M. Feng, N. A. Brilliant, S. T. Cundiff, R. P. Mirin, and K. L. Silverman, IEEE Photon. Technol. Lett. 19, 804 (2007).

${ }^{6}$ Y. Chu, M. G. Thompson, R. V. Penty, I. H. White, and A. R. Kovsh, CLEO/QELS OSA Technical Digest (Optical Society of America, Washington, DC, 2007), p. CMP4.

${ }^{7}$ D. J. H. C. Maas, A. R. Bellancourt, M. Hoffman, B. Rudin, Y. Barbarin, M. Golling, T. Sudmeyer, and U. Keller, Opt. Express 16, 18646 (2008).

${ }^{8}$ T. C. Newell, D. J. Bossert, A. Stintz, B. Fuchs, K. J. Malloy, and L. F. Lester, IEEE Photon. Technol. Lett. 11, 1527 (1999).

${ }^{9}$ G. Visimberga, G. Rainò, A. Salhi, V. Tasco, M. T. Todaro, L. Martiradonna, M. De Giorgi, A. Passaseo, R. Cingolani, and M. De Vittorio, Appl. Phys. Lett. 93, 151112 (2008).

${ }^{10}$ K. L. Hall, G. Lenz, E. P. Ippen, and G. Raybon, Opt. Lett. 17, 874 (1992).

${ }^{11}$ D. B. Malins, A. Gomez-Iglesias, S. J. White, W. Sibbett, A. Miller, and E. U. Rafailov, Appl. Phys. Lett. 89, 171111 (2006).

${ }^{12}$ T. Piwonski, J. Pulka, G. Madden, G. Huyet, J. Houlihan, E. A. Viktorov, T. Erneux, and P. Mandel, Appl. Phys. Lett. 94, 123504 (2009).

${ }^{13}$ I. O’Driscoll, T. Piwonski, J. Houlihan, G. Huyet, R. J. Manning, and B. Corbett, Appl. Phys. Lett. 91, 263506 (2007). 